$$
\text { "zeitler2" — 2005/2/16 — 19:56 — page 209 — \#1 }
$$

\title{
Packings in hyperbolic geometry
}

\author{
H. ZEITLER
}

Abstract. I am becoming older. That's why I am returning to my youth sins. "On revient toujours à ses premiers amoures". This sin was the noneuclidean hyperbolic geometry - especially the Poincaré model. I was teaching this kind of geometry over many years as well in highschool (Gymnasium) as for beginners at the university too.

A lot of results concerning packings in hyperbolic geometry are proved by the Hungarian school around László Fejes Tóth. In this paper we construct very special packings and investigate the corresponding densities. For better understanding we are working in the Poincaré model. At first we give a packing of the hyperbolic plane with horodisks and calculate the density. In an analogous way then the hyperbolic space is packed by horoballs. In the last case the calculation of the density is a little bit difficult. Finally it turns out that in both cases the maximal density is reached.

Key words and phrases: hyperbolic geometry, packing, covering, partitioning, density.

ZDM Subject Classification: G90.

\section{Introduction}

\subsection{Some definitions}

Let $E_{i}$ be a family of given point sets and $D$ a domain. If $\cup E_{i} \subset D$ and no two sets $E_{i}, E_{j}$ have common points (up to boundary elements) then we speak about a packing of $D$. Naturally in this case gaps may occur. Think of a box $D$ filled with oranges $E_{i}$.

Copyright (C) 2004 by University of Debrecen
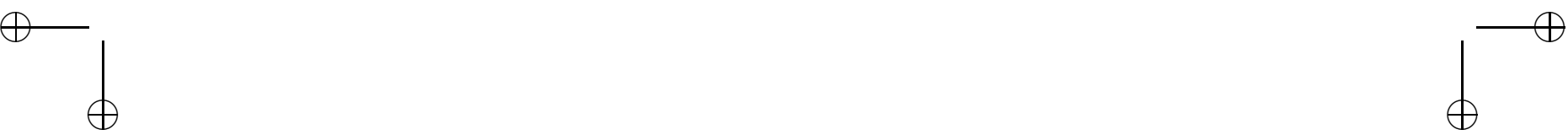


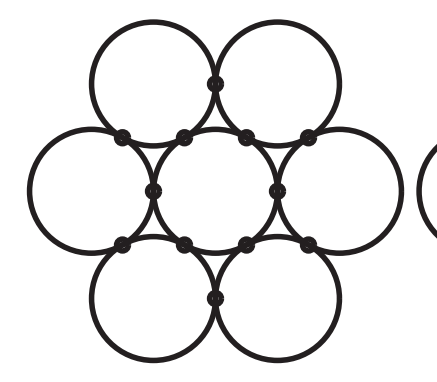

packing

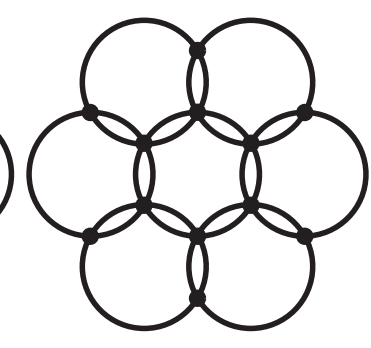

covering

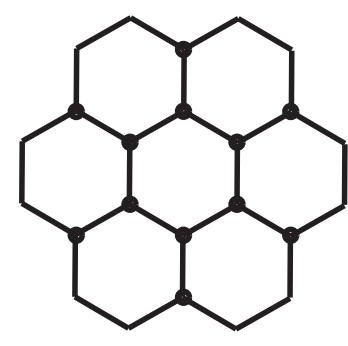

partitioning

Figure 1. Packing, covering, partitioning

Density

We are working with the usual definition of the density due to L. Fejes Tóth:

$$
d=\frac{\sum_{i} I\left(E_{i}\right)}{I(D)}
$$

$I\left(E_{i}\right)$ and $I(D)$ means the content of $E_{i}$ respectively of $D$. In the plane this is the area and in space the volume. Then a problem is to find packings with maximal density.

\subsection{Aim of the paper}

We restrict ourselves to considerations in the hyperbolic plane $\mathbb{H}^{2}$ and in the hyperbolic space $\mathbb{H}^{3}$. All investigations are done within the Poincaré halfplane or halfspace model. Using congruent hyperbolic disks and congruent hyperbolic balls (with radius $r$ each) L. Feher Tóth obtained the following results for packings

$$
\mathbb{H}^{2}: d(r)<\frac{3}{\pi} \approx 0,9549, \quad \mathbb{H}^{3}: d(r)<\frac{2}{3 A} \approx 0,852
$$

A is the so-called Coxeter series $A=\sum_{K=0}^{\infty}\left[\frac{1}{(1+3 K)^{2}}-\frac{1}{(2+3 K)^{2}}\right]$. We are interested only in the limiting case.

These limits of density cannot be attended by disks and balls, rather it needs so-called horodisks or horoballs.

Remark concerning notions:

In Euclidean plane the set of points $P$ with $\overline{P M}=r$ is called circle with center $M$ and radius $r$ and the set $\overline{P M} \leqq r$ the corresponding disk. In an analogous 


$$
\text { "zeitler2" — 2005/2/16 — 19:56 — page 211 — \#3 }
$$

way we distinguish between sphere and ball in Euclidean space. We transfer all these notions to hyperbolic geometry.

\section{A packing of the hyperbolic plane $\mathbb{H}^{2}$}

\subsection{The Poincaré halfplane model}

We give only a sketch and refer to special books (see e.g. [14]). The elements of Euclidean geometry are supplied with new name plates, with new etiquettes. Some geometers are speaking about a translation from Euclidean to hyperbolic language - they use a dictionary.

For better distinction we write $E, H$ for the respective notions: $H$-line, $E$ circle, ...

$H$-points:

The set of all $E$-points in a distinguished open $E$-halfplane. The limiting line is $x$.

Ideal $H$-points:

The set of all $E$-points in $x$ and one point at infinity $P_{\infty}$.

$H$-lines:

The set of all $E$-circles and $E$-lines orthogonal to $x$ - as far as they are in the distinguished halfplane.

Horocycles:

The set of all $E$-circles touching $x$, together with the set of all $E$-lines parallel to $x$, as far they are in distinguished halfplane.

Exactly as in the remark to 1.2 we naturally have also horodisks.

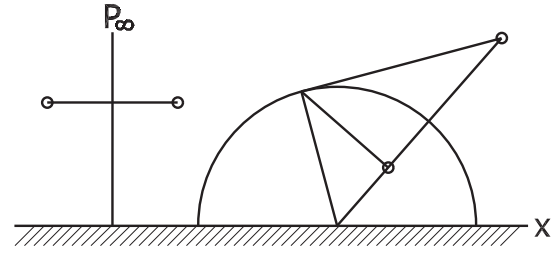

$H$-lines and $H$-reflections

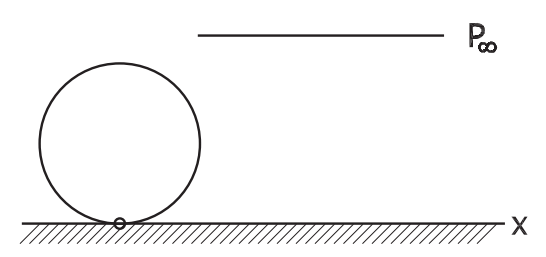

horocycles

Figure 2. $H$-lines, $H$-reflections, horocycles 


$$
\text { "zeitler2" — 2005/2/16 — 19:56 — page 212 — \#4 }
$$

$H$-reflection in $H$-lines:

$E$-reflection in the $E$-circle (inversion) or in the $E$-line corresponding to the $H$-line in question.

$H$-angles:

$H$-angles are the corresponding $E$-angles. The degrees of $H$-angles are measured in the Euclidean way.

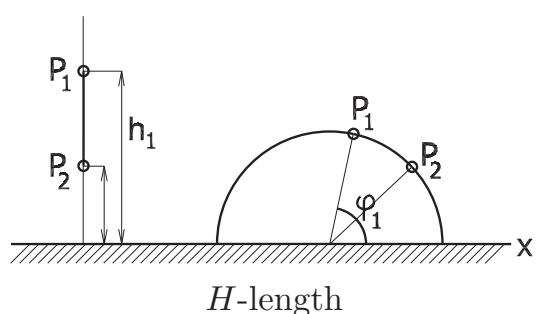

$H$-length

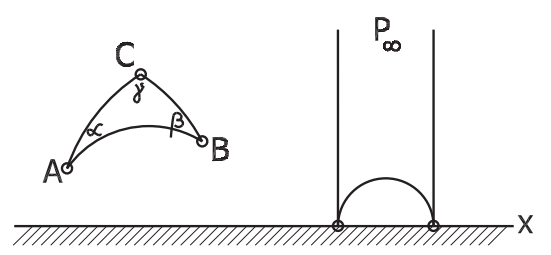

$H$-area

$$
\begin{aligned}
& \overline{P_{1} P_{2}}=\ln \frac{h_{1}}{h_{2}} \quad \overline{P_{1} P_{2}}=\frac{1}{2} \ln \frac{1+\cos \varphi_{1}}{1-\cos \varphi_{1}}: \frac{1+\cos \varphi_{2}}{1-\cos \varphi_{2}} \\
& F=\pi-(\alpha+\beta+\gamma)
\end{aligned}
$$

Figure 3. $H$-length, $H$-area $F$

Now it's possible to develop all the flora and fauna of twodimensional hyperbolic geometry within the model.

$H$-distance, $H$-area, $H$-trigonometry, $\ldots$

$H$-theorems as for instance: $H$-distance, $H$-angle and $H$-area remain invariant under $H$ reflections.

\subsection{The construction of the packing}

\subsubsection{The first step}

What can we see in Figure 6?

In E-language:

Two lines $g_{1}, g_{2}$ orthogonal to $x$ in $M_{1}, M_{2}$ two $E$-circles $H_{1}, H_{2}$ touching one another and touching $x$ in $M_{1}, M_{2}$. One line $H_{0}$ touching our two $E$-circles in $A_{1}, A_{2}$ and parallel to $x$. Finally one $E$-circle over $M_{1}, M_{2}$ orthogonal to $x$.

We consider only the parts of all these curves within the $E$-strip formed by $g_{1}, g_{2}$ and $x$. 


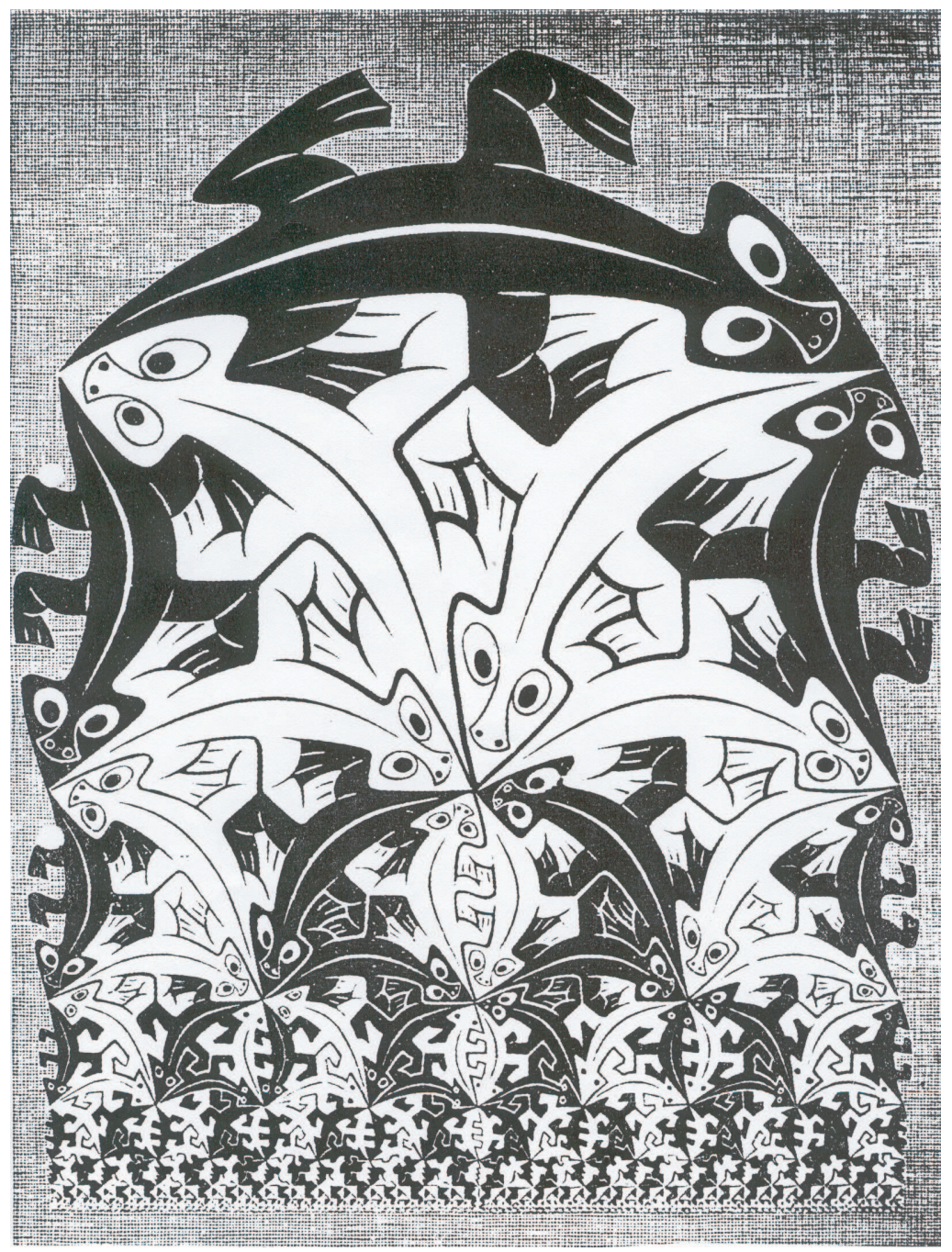

Figure 4. Woodcut due to M. C. Escher

In $H$-language:

$g_{1}, g_{2}$ and the $E$-circle over $M_{1}, M_{2}$ are $H$-lines. They form the $H$-triangle $D$ with vertices $M_{1}, M_{2}, P_{\infty}$. All these vertices are ideal points. The measure of the angles in this triangle is 0 each. We are speaking about a 3 -fold asymptotic $H$-triangle. Further we see three horocycle arcs. 


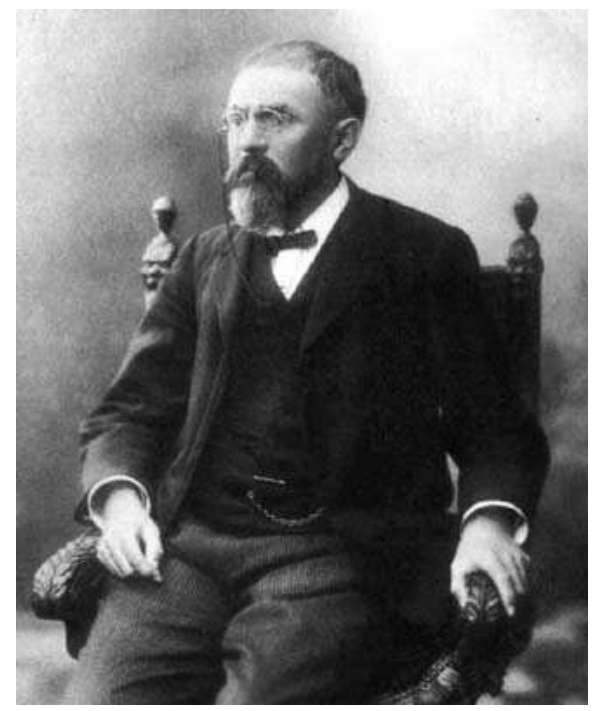

Figure 5. Henri Poincaré (1854-1912)

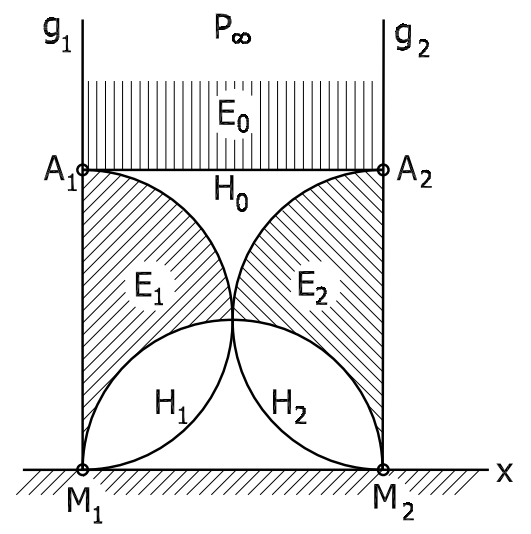

Figure 6. The first step

In this way we obtain three horodisk sectors - denoted by $E_{0}, E_{1}, E_{2}$. Each bounded by two $H$-segments and one horocycle arc. The 3 -fold asymtotic triangle is packed by these three horodisk sectors. Between, there remains a gap, a triangle formed by three horocycle arcs. We have no overlapping. 


$$
\text { "zeitler2" — 2005/2/16 — 19:56 — page 215 — \#7 }
$$

\subsubsection{The second step}

In Figure 7 we now perform a $H$-reflection in the $H$-line through $M_{1}, M_{2}$. Doing so the 3 -fold asymptotic $H$-triangle $\left(M_{1}, M_{2}, P_{\infty}\right)$ is mapped into another $H$ triangle $\left(M_{1}, M_{2}, M_{3}\right)$ of the same kind. The horocycles $H_{1}, H_{2}$ remain fixed whilst $H_{0}$ is becoming a new horocycle. Our horodisk sectors $E_{0}, E_{1}, E_{2}$ are mapped in new horodisk sectors. The area remains in any case invariant. The new $H$-triangle is packed by these disk sectors. Between, there remains again a gap, a triangle formed by three horocycle arcs.

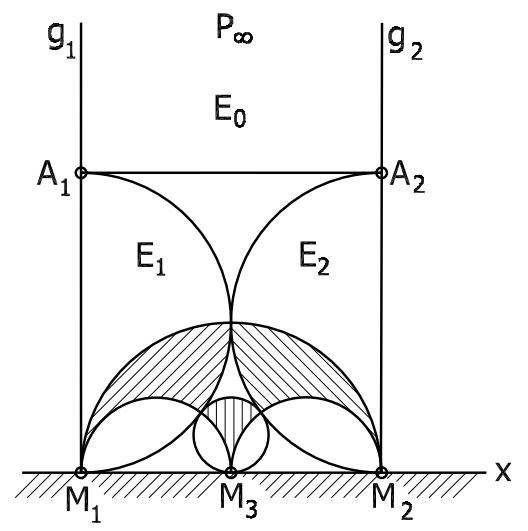

Figure 7. The second step

\subsubsection{We continue!}

In Figure 7 we now perform a $H$-reflection in the $H$-line through $M_{1}, M_{3}$. Doing so we obtain again a 3 -fold asymtotic $H$-triangle $\left(M_{1}, M_{4}, M_{3}\right)$ packed by three horodisk sectors. We repeat this procedure again and again. New 3-fold asymptotic $H$-triangles are born, each packed by three horodisk sectors always with the same area. Finally the strip formed by $g_{1}, g_{2}$ is totally packed. Reflection in the $H$-lines $g_{1}, g_{2}$ yields one packed strip after the next. In this way we receive a packing of the total plane $\mathbb{H}^{2}$. Figure 8 gives an idea.

\subsection{Density}

We first give and prove a very surprising lemma - the so-called BolyaiLobachevsky theorem on horodisk sectors. 


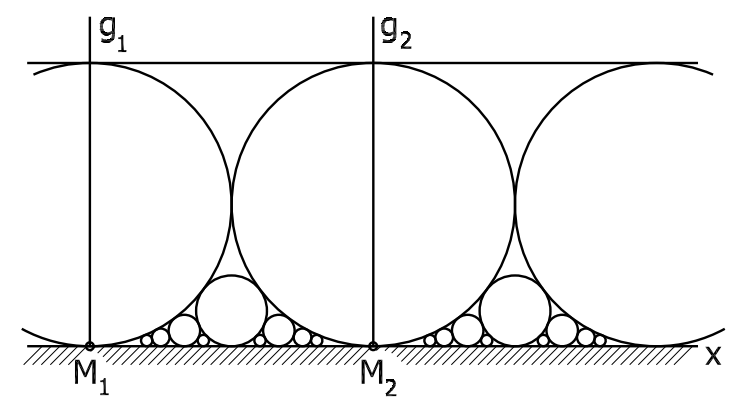

Figure 8. From strip to $\mathbb{H}^{2}$

\subsubsection{Lemma}

The $H$-length $b$ of the horocycle arc $A_{1} A_{2}$ in Figure 6 is exactly the area $F\left(E_{0}\right)$ of the corresponding horodisk sector with vertices $A_{1}, A_{2}, P_{\infty}$.

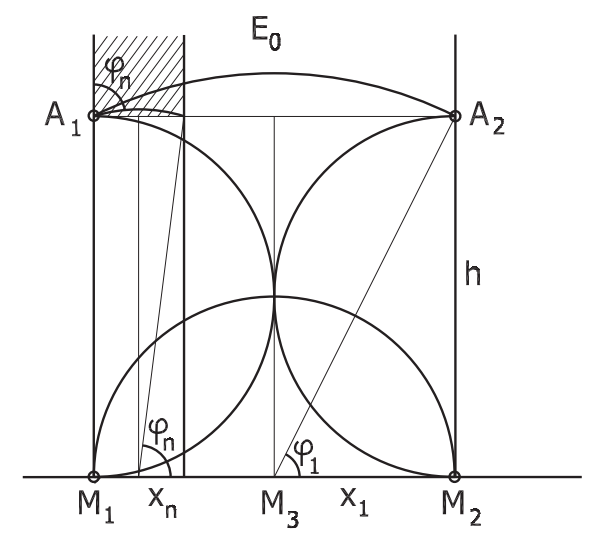

Figure 9. $F\left(E_{0}\right)=2 \operatorname{sh} \frac{a_{1}}{2}$

Proof.

(a) Two formula

A first formula

Due to the definition in Figure 3 and with the notations from Figure 9 we have

$$
a_{1}=\frac{1}{2} \ln \frac{1+\cos \varphi_{1}}{1-\cos \varphi_{1}}: \frac{1-\cos \varphi_{1}}{1+\cos \varphi_{1}}=\ln \frac{1+\cos \varphi_{1}}{1-\cos \varphi_{1}}
$$




$$
\text { "zeitler2" — 2005/2/16 — 19:56 — page } 217 \text { — \#9 }
$$

$$
\begin{aligned}
& \Rightarrow \frac{a_{1}}{2}=\ln \sqrt{\frac{1+\cos \varphi_{1}}{1-\cos \varphi_{1}}} \Rightarrow e^{\frac{a_{1}}{2}}=\sqrt{\frac{1+\cos \varphi_{1}}{1-\cos \varphi_{1}}} \\
& \Rightarrow \operatorname{sh} \frac{a_{1}}{2}=\frac{1}{2}\left(e^{\frac{a_{1}}{2}}-e^{-\frac{a_{1}}{2}}\right)=\frac{1}{2} \cdot \frac{2 \cos \varphi_{1}}{\sin \varphi_{1}}=\operatorname{ctg} \varphi_{1}=\frac{x_{1}}{h} .
\end{aligned}
$$

A second formula

From $e^{a_{1}}=\frac{1+\cos \varphi_{1}}{1-\cos \varphi_{1}}$ we obtain $\cos \varphi_{1}=\frac{e^{a_{1}}-1}{e^{a_{1}}+1}=\operatorname{th} \frac{a_{1}}{2}$

(b) Halving

The $E$-segment $x_{1}$ in Figure 9 is halved again and again. In the step of number $n$ bisection yields $2^{n} E$-segments of $E$-length $x_{n}=\frac{x_{1}}{2^{n-1}}$. Further there are $2^{n-1}$ corresponding $H$-segments of $H$-length $a_{n}$.

(c) Extension

By induction the two formula in (a) can be extended. We receive

$$
\operatorname{sh} \frac{a_{n}}{2}=\operatorname{ctg} \varphi_{n}=\frac{x_{n}}{h}=\frac{x_{1}}{2^{n-1} h}=\frac{1}{2^{n-1}} \operatorname{sh} \frac{a_{1}}{2} \quad \text { and } \quad \cos \varphi_{n}=\operatorname{th} \frac{a_{n}}{2} .
$$

(d) Horocycle arc

We perform a limiting process. $n$ is running to infinity.

$$
b=\lim _{n \rightarrow \infty} 2^{n-1} a_{n}=\lim _{n \rightarrow \infty} 2^{n}\left(\frac{\frac{a_{n}}{2}}{\operatorname{sh} \frac{a_{n}}{2}}\right) \operatorname{sh} \frac{a_{n}}{2}=\lim _{n \rightarrow \infty} 2^{n} A_{n} \frac{1}{2^{n-1}} \operatorname{sh} \frac{a_{1}}{2}=2 \operatorname{sh} \frac{a_{1}}{2} .
$$

We used $\lim _{n \rightarrow \infty} A_{n}=1$ and $\operatorname{sh} \frac{a_{n}}{2}=\frac{1}{2^{n-1}} \operatorname{sh} \frac{a_{1}}{2}$.

(e) Horodisk sector

Once more $n$ is running to infinity.

The area of the marked small $H$-triangle with angles $0, \varphi_{n}, \varphi_{n}$ in Figure 9 is $\pi-2 \varphi_{n}-$ due to the formula given in Figure 3. With this we obtain

$$
\begin{aligned}
F\left(E_{0}\right) & =\lim _{n \rightarrow \infty} 2^{n-1}\left(\pi-2 \varphi_{n}\right)=\lim _{n \rightarrow \infty} 2^{n-1} \frac{\pi-2 \varphi_{n}}{\sin \left(\pi-2 \varphi_{n}\right)} \sin \left(\pi-2 \varphi_{n}\right) \\
& =\lim _{n \rightarrow \infty} 2^{n-1} A_{n} \sin 2 \varphi_{n}=\lim _{n \rightarrow \infty} 2^{n} A_{n} \sin \varphi_{n} \cos \varphi_{n} \\
& =\lim _{n \rightarrow \infty} 2^{n} A_{n} \sin \varphi_{n} \text { th } \frac{a_{n}}{2}=\lim _{n \rightarrow \infty} 2^{n} A_{n} \sin \varphi_{n} \frac{\operatorname{sh} \frac{a_{n}}{2}}{\operatorname{ch} \frac{a_{n}}{2}} \\
& =\lim _{n \rightarrow \infty} 2^{n} A_{n} \sin \varphi_{n} \frac{1}{\operatorname{ch} \frac{a_{n}}{2}} \cdot \frac{1}{2^{n-1}} \operatorname{sh} \frac{a_{1}}{2}=2 \operatorname{sh} \frac{a_{1}}{2} .
\end{aligned}
$$

We used the extended formula in (c) further:

$$
\lim _{n \rightarrow \infty} A_{n}=1, \lim _{n \rightarrow \infty} \varphi_{n}=\frac{\pi}{2}, \lim _{n \rightarrow \infty} \sin \varphi_{n}=1, \lim _{n \rightarrow \infty} \operatorname{ch} \frac{a_{n}}{2}=1,
$$


Now we are going back to the density.

Because of our special construction it is enough to calculate the density in only one 3 -fold asymptotic $H$-triangle - we choose the $H$-triangle $\left(M_{1}, M_{2}, P_{\infty}\right)$. Due to our reasonable definition we have $d=\frac{F\left(E_{0}\right)+F\left(E_{1}\right)+F\left(E_{2}\right)}{F(D)}$. This will be the density of our packing all over the hyperbolic plane $\mathbb{H}^{2}$.

\subsubsection{Theorem}

The density of our packing the hyperbolic plane $\mathbb{H}^{2}$ with horodisks is $d=\frac{3}{\pi} \approx$ 0,9549 .

With this we can say that the upper bound density given by L. Fejes Tóth is reached.

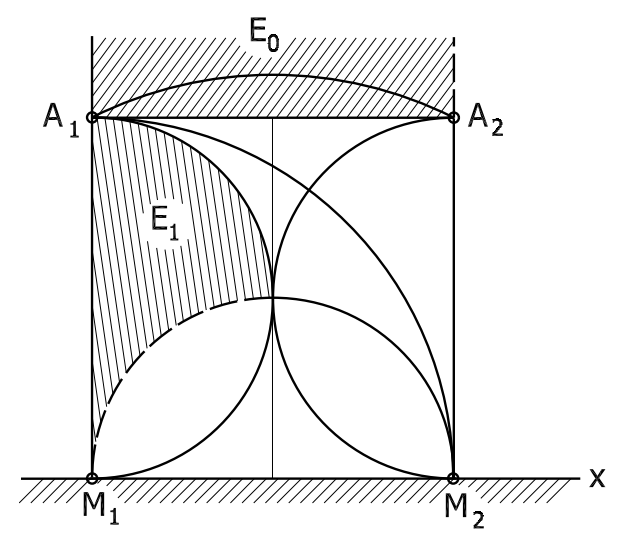

Figure 10. $E_{0} \rightarrow E_{1}$

Proof. Due to Figure 3 with $\alpha=\beta=\gamma=0$ we have $F(D)=\pi$. To find $F\left(E_{0}\right)$ we need our Lemma 2.3.1 and obtain $F\left(E_{0}\right)=2 \operatorname{sh} \frac{a_{1}}{2}=2 \frac{x_{1}}{h}$ and with $h=2 r, x_{1}=r$ finally $F\left(E_{0}\right)=1$.

Finding $F\left(E_{1}\right)$ we use a trick. We perform a $H$-reflection in the $H$-line through $M_{2}$ and $A_{1}$ (Figure 10 ). The corresponding $E$-circle has center $M_{1}$ and radius $2 r$. Doing so $H_{0}$ is mapped to $H_{1}$ and $g_{2}$ to the $H$-line over $M_{1}, M_{2}$. The $H$-line $g_{1}$ remains as a whole. So we can say that the horodisk sector $E_{0}$ is mapped on $E_{1}$.

$H$-reflection preserves area, therefore $F\left(E_{1}\right)=F\left(E_{0}\right)$. 


$$
\text { "zeitler2" — 2005/2/16 — 19:56 — page } 219 \text { — \#11 }
$$

In the same way we obtain $F\left(E_{2}\right)=F\left(E_{0}\right)$. Finally we have

$$
d=\frac{F\left(E_{0}\right)+F\left(E_{1}\right)+F\left(E_{2}\right)}{F(D)}=\frac{3}{\pi} .
$$

\section{A packing of the hyperbolic space}

\subsection{The Poincaré halfspace model}

We give a few "etiquettes" and refer to the model of $\mathbb{H}^{2}$.

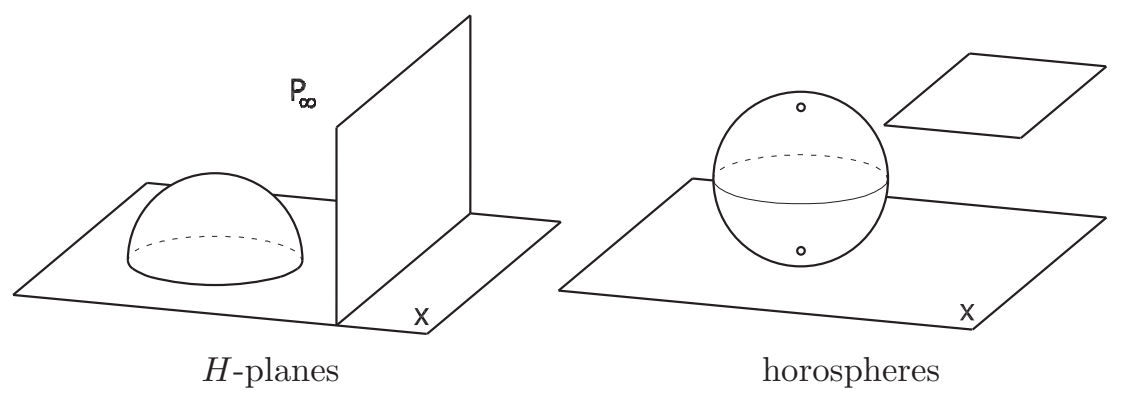

Figure 11. H-planes, horospheres

$H$-points:

The set of all $E$-points in a distinguished open $E$-halfspace. The limiting plane is $x$.

Ideal points:

The set of all $E$-points in $x$ and one point at infinity $P_{\infty}$.

$H$-planes:

The set of all $E$-spheres and $E$-planes orthogonal to $x$ - as far as they are in the distinguished halfspace.

$H$-lines:

The set of all $E$-circles and $E$-lines in our halfspace orthogonal to $x$.

Horospheres:

The set of all $E$-spheres touching $x$, together with the set of all $E$-planes parallel to $x$ - as far as they are in the distinguished halfspace.

Exactly as in the remark to 1.2 we naturaly have also horoballs. 


$$
\text { "zeitler2" — 2005/2/16 — 19:56 — page 220 — \#12 }
$$

$H$-reflection in $H$-planes:

$E$-reflection in the $E$-sphere (inversion) or in the $H$-plane corresponding to the $H$-plane in question.

$H$-angle:

$H$-angles are the corresponding $E$-angles. The degrees of $H$-angles are measured in the Euclidean way.

Exactly as in the twodimensional case now it's possible to develop the flora and fauna of threedimensional hyperbolic geometry within the model. We recommend the reader to do this and to use older books. Mathematics must be done!

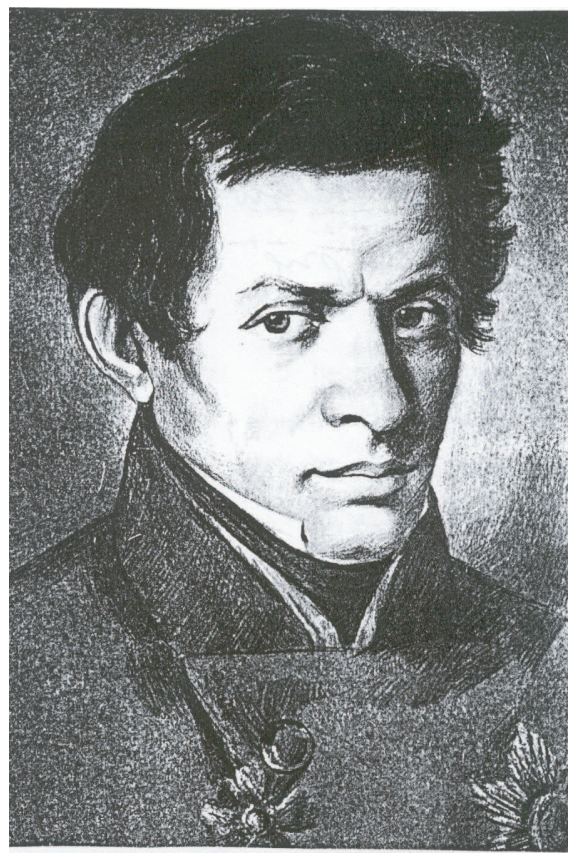

Figure 12. Nikolai Ivanovich Lobachevsky (1792-1856) 


$$
\text { "zeitler2" — 2005/2/16 — 19:56 — page } 221 \text { — \#13 }
$$

\subsection{The construction of the packing}

\subsubsection{The first step}

In $E$-language:

We start with an equilateral $E$-triangle $\left(M_{1}, M_{2}, M_{3}\right)$ in $x$ (length of one edge is $2 r$ ) and three $E$-lines $g_{1}, g_{2}, g_{3}$ in $M_{1}, M_{2}, M_{3}$ orthogonal to $x$. So we have a prism with faces $S_{1}, S_{2}, S_{3}$. Further we draw a $E$-sphere $H$ (radius $\frac{2}{3} r \sqrt{3}$ ) through $M_{1}, M_{2}, M_{3}$ also orthogonal to $x$ and consider the domain $D$ included by the faces of the prism and the $E$-sphere $H$ (Figure 13).

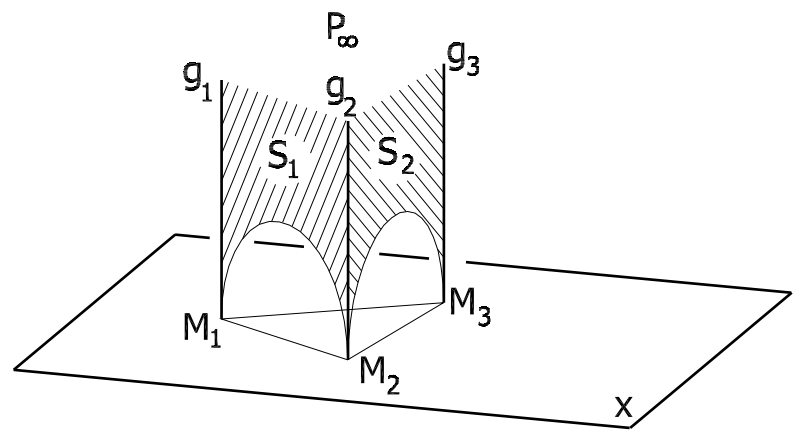

Figure 13. 4-fold-asymptotic $H$-tetrahedron

Translation in $H$-language:

The points $M_{1}, M_{2}, M_{3}, P_{\infty}$ form a 4 -fold asymptotic $H$-tetrahedron. Because the triangle $\left(M_{1}, M_{2}, M_{3}\right)$ is equilateral we are speaking about a regular $H$-tetrahedron. The faces are 3 -fold asymptotic $H$-triangles.

The construction now is continued using again the $E$-language:

We draw three $E$-spheres respectively three $E$-balls $H_{1}, H_{2}, H_{3}$ radius $r$ touching $x$ in the points $M_{1}, M_{2}, M_{3}$. Then we consider only the ball sectors within the prism. Think of orange slices. These ball sectors are touching one another.

The $E$-plane parallel to $x$ touching $H_{1}, H_{2}, H_{3}$ in the points $A_{1}, A_{2}, A_{3}$ is denoted by $H_{0}$.

The $E$-sphere $H$ cuts all the sectors and so we obtain truncated horoball sectors.

Back to the $H$-language:

We denote the three truncated sectors $E_{1}, E_{2}, E_{3}$ now as horoball sectors - each bounded by three $H$-planes and one horosphere (Figure 15). The part of the $E$-prism over the $E$-triangle $\left(A_{1}, A_{2}, A_{3}\right)$ is also bounded by three $H$-planes and 


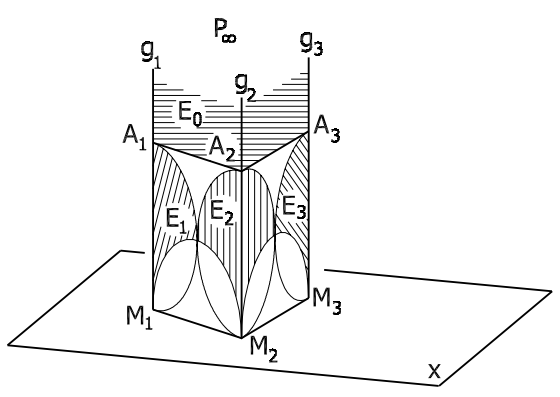

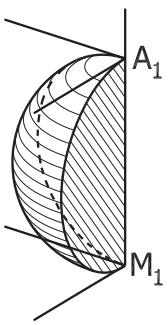

one slice

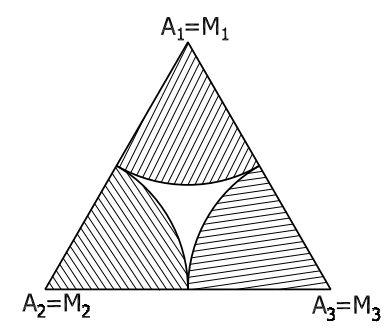

top view of 3 slices

Figure 14. Horosphere - slices

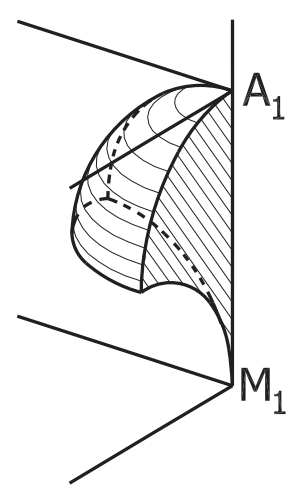

Figure 15. One "truncated" slice

one horosphere. Therefore we have another truncated horoball sector $E_{0}$. With our definition in the introduction we have a packing of our 4-fold asymptotic $H$ tetrahedron with four truncated horoball sectors. There is no overlapping, but one gap occours (Figure 14).

\subsubsection{The second step}

If a geometrical configuration was difficult to understand, then the famous geometer Jakob Steiner (1796-1863) from Switzerland told his students to close the eyes. Or he even darkened the classroom - for better seeing, for better visualizing geometrical objects. Now our construction is arrived at a point, where we should do in this way. 


$$
\text { "zeitler2" — 2005/2/16 — 19:56 — page 223 — \#15 }
$$

The construction works in a similar way as in the plane case. We can take over some texts word-by-word.

We perform a $H$-reflection in the $H$-plane $H$. Doing so the 4 -fold asymptotic tetrahedron $\left(M_{1}, M_{2}, M_{3}, P_{\infty}\right)$ is mapped into another tetrahedron $\left(M_{1}, M_{2}, M_{3}\right.$, $\left.M_{4}\right)$ of the same kind. $M_{4}$ is the $E$-center of the $E$-triangle $\left(M_{1}, M_{2}, M_{3}\right)$. The horospheres $H_{1}, H_{2}, H_{3}$ remain fixed as a whole, whilst $H_{0}$ is becoming a new horosphere $H_{4}$ (the corresponding $E$-sphere touches $x$ in $M_{4}$, radius $\frac{1}{3} r$ ). The $H$-planes $S_{1}, S_{2}, S_{3}$ are going to $H$-planes through $M_{4}$ and $M_{1} M_{2}, M_{1} M_{3}$ or $M_{2} M_{3}$ respectively.

Figure 16 shows the situation from a top view.
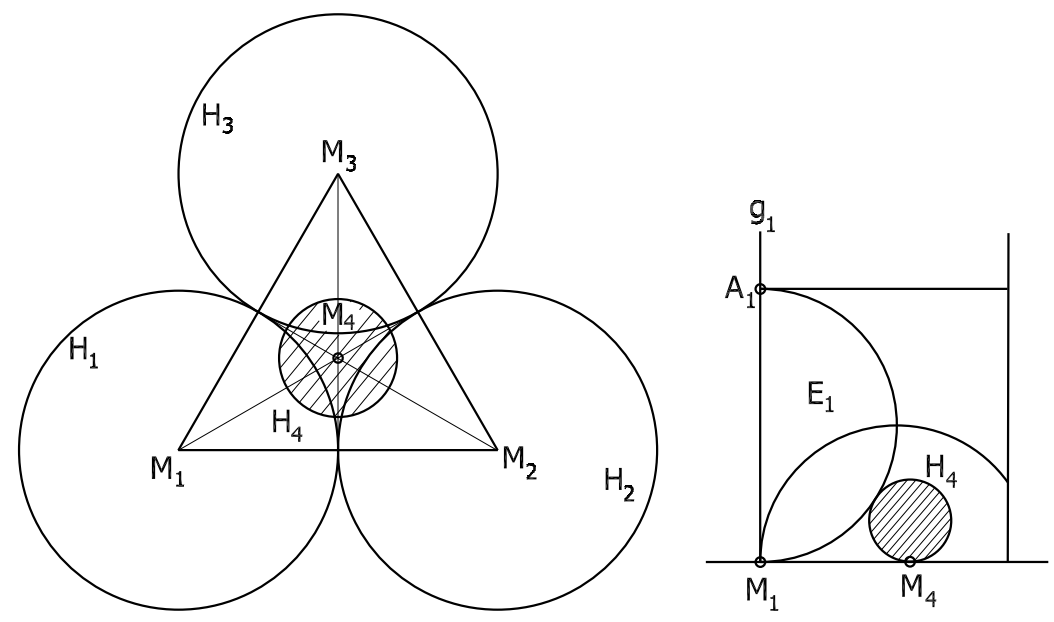

Figure 16

The pictures of our horoball sectors $E_{1}, E_{2}, E_{3}, E_{0}$ are new ones - each bounded by three $H$-planes and one horosphere (remember J. Steiner!) The volume remains invariant in any case. The new 4-fold asymptotic $H$-tetrahedron is packed by these horoball sectors. No overlapping, but a gap occurs.

\subsubsection{We continue}

Now we perform a $H$-reflection in the $H$-plane through $M_{1}, M_{2}, M_{4}$. Doing so, we obtain a 4 -fold asymptotic $H$-tetrahedron $\left(M_{1}, M_{2}, M_{4}, M_{5}\right)$ packed with four horoball sectors. 


$$
\text { "zeitler2" — 2005/2/16 — 19:56 — page } 224 \text { — \#16 }
$$

We repeat this procedure again and again. New 4-fold asymptotic $H$-tetrahedra are born, each packed by four horoball sectors. Always with the same volume.

Finally the prism formed by $g_{1}, g_{2}, g_{3}$ is totally packed.

$H$-reflections in the $H$-planes $S_{1}, S_{2}, S_{3}$ yield one packed prism after the next.

In this way we receive a packing of the space $\mathbb{H}^{3}$.

\subsection{Density}

The so-called Bolyai-Lobachevsky theorem works not only in the case of dimension 2 but with some modifications also in higher dimensions.

\subsubsection{Lemma}

The $H$-volume of the horoball sector $E_{0}$ with vertices $A_{1}, A_{2}, A_{3}, P_{\infty}$ is exactly half of the $H$-area $F$ of the horosphere triangle with vertices $A_{1}, A_{2}, A_{3}$.

In short $V\left(E_{0}\right)=\frac{1}{2} F$.

The proof is done using the so-called "volume element". We omit this proof here and refer for instance to [6].

Now back to the density

\subsubsection{Theorem}

The density of our packing the hyperbolic space $\mathbb{H}^{3}$ with horoballs is $d=\frac{2}{3 A}$ with $A=\sum_{K=0}^{\infty}\left[\frac{1}{(1+3 K)^{2}}-\frac{1}{(2+3 K)^{2}}\right]$

PROOF. Because of our very special construction it is enough to calculate the density in one 4-fold asymptotic $H$-tetrahedron only. We choose the tetrahedron $\left(M_{1}, M_{2}, M_{3}, P_{\infty}\right)$. Due to our definition in the introduction we then have

$$
d=\frac{\sum_{i=0}^{3} V\left(E_{i}\right)}{V(D)} .
$$

This is the density of our packing the total hyperbolic space $\mathbb{H}^{3}$ too.

The proof is done in several steps.

The volume of the 4-fold asymptotic regular tetrahedron $V(D)$.

One possibility for calculation is to use once more the "volume element" [6] together with a decomposition into six orthoschemes [1]. Applying these two 
instruments in the Poincaré halfspace model we obtain

$$
V(D)=3 \sqrt{3} \int_{0}^{\infty} \frac{x}{e^{2 x}+e^{-2 x}+1} d x .
$$

This result is given and proved already by N. I. Lobachevsky [7].

The substitution $z=2 x$ gives $V(D)=\frac{3}{4} \sqrt{3} \int_{0}^{\infty} \frac{z}{e^{z}+e^{-z}+1} d z$. Developing the integrand in a series and then performing integration by parts we obtain

$$
\begin{aligned}
V(D) & =\frac{3}{4} \sqrt{3} \int_{0}^{\infty} z \sum_{K=0}^{\infty}\left[e^{-2(1+3 K)}-e^{-2(2+3 K)}\right] \\
& =\frac{3}{4} \sqrt{3} \sum_{K=0}^{\infty}\left[\frac{1}{(1+3 K)^{2}}-\frac{1}{(2+3 K)^{2}}\right]=\frac{3}{4} \sqrt{3} \cdot A
\end{aligned}
$$

The volume $V\left(E_{0}\right)$ of the horoball sector $E_{0}$

Referring to our Lemma 3.3.1 it's enough to calculate $F$.

This is done by approximation in nearly the same way as in the chapter about $\mathbb{H}^{2}$. Some formulations are equivalent.

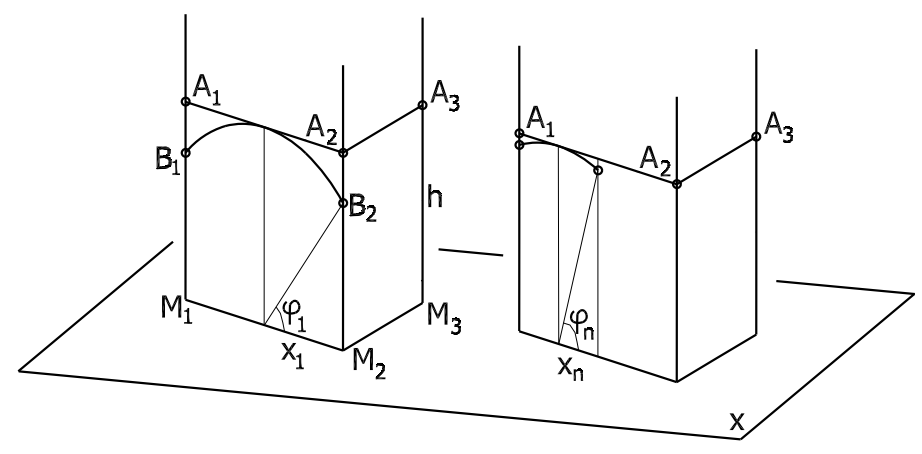

Figure 17

(a) Two formula

We use the results in 2.3.1 in respect of one face of our prism.

Let the length of the $H$-segment $B_{1} B_{2}$ in Figure 17 be $b_{1}$. Then we have

$$
\operatorname{sh} \frac{b_{1}}{2}=\operatorname{ctg} \varphi_{1} \quad \text { and } \quad \text { th } \frac{b_{1}}{2}=\cos \varphi_{1}=\frac{x_{1}}{h} .
$$

Attention:

In 2.3.1 the $E$-segment $A_{1} A_{2}$ was approximated by $H$-segments from above (Figure 9) and now from below. 
(b) Halving

The $E$-segment $x_{1}$ is now halved again and again. In step of number $n$ this bisection yields $2^{n} E$-segments of length $x_{n}=\frac{x_{1}}{2^{n-1}}$. Further there are $2^{n-1}$ corresponding $H$-segments of length $b_{n}$

(c) Extension

The formula in (a) can be extended by induction.

$$
\operatorname{sh} \frac{b_{n}}{2}=\operatorname{ctg} \varphi_{n} \quad \text { and } \quad \operatorname{th} \frac{b_{n}}{2}=\cos \varphi_{n}=\frac{x_{n}}{h}=\frac{x_{1}}{2^{n-1} h}=\frac{1}{2^{n-1}} \text { th } \frac{b_{1}}{2}
$$

(d) Approximation of $F$

Now we consider $E$-triangles in $\left(M_{1}, M_{2}, M_{3}\right)$ built by $E$-segments of length $x_{n}$ and $H$-triangles touching $\left(A_{1}, A_{2}, A_{3}\right)$ from below with $H$-segments $b_{n}$. Figure 18 shows the situation in case $n=2$. All these $H$-triangles have a shape like a stepped blanket. The number of such equilateral $H$-triangles in step of number $n$ is $4^{n-1}$.

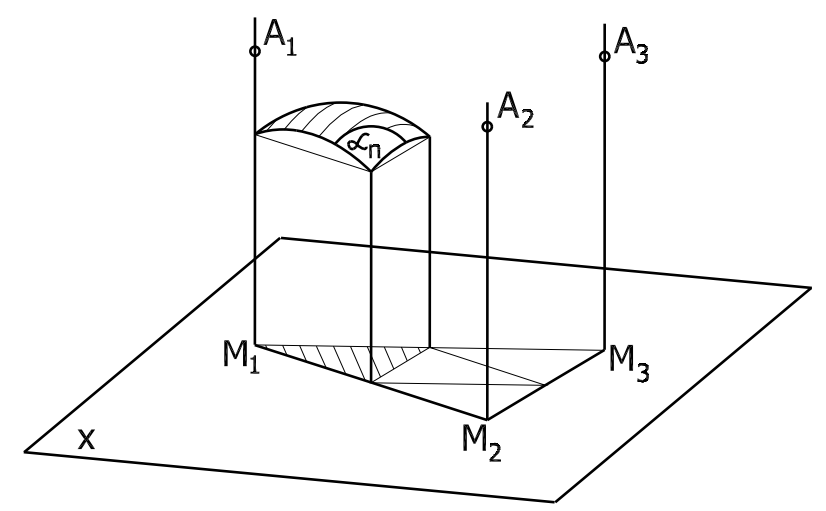

Figure 18

(e) Limiting process

$$
\begin{aligned}
F & =\lim _{n \rightarrow \infty} 4^{n-1}\left(\pi-3 \alpha_{n}\right) \\
& =\lim _{n \rightarrow \infty} 4^{n-1}\left(\frac{\pi-3 \alpha_{n}}{\sin \left(\pi-3 \alpha_{n}\right)}\right) \sin \left(\pi-3 \alpha_{n}\right) \\
& =\lim _{n \rightarrow \infty} 4^{n-1} A_{n} \sin 3 \alpha_{n}
\end{aligned}
$$


Using addition theorems it's easy to prove $\sin 3 \alpha_{n}=\sin \alpha_{n}\left(4 \cos ^{2} \alpha_{n}-1\right)$. For hyperbolic functions we get also an addition theorem cth $b_{n}=\frac{1+\operatorname{th}^{2} \frac{b_{n}}{2}}{2 \operatorname{th} \frac{b_{n}}{2}}$. From one equilateral $H$-triangle with edges of length $b_{n}$ we deduce with classical $H$ trigonometry $\cos \alpha_{n}=\operatorname{cth} b_{n} \cdot$ th $\frac{b_{n}}{2}$. Altogether this yields

$$
\begin{gathered}
\cos \alpha_{n}=\operatorname{cth} b_{n} \operatorname{th} \frac{b_{n}}{2}=\frac{1}{2}\left(1+\operatorname{th}^{2} \frac{b_{n}}{2}\right) \\
4 \cos ^{2} \alpha_{n}-1=\frac{4\left(1+\operatorname{th}^{2} \frac{b_{n}}{2}\right)^{2}}{4}-1=2 \operatorname{th}^{2} \frac{b_{n}}{2}+\operatorname{th}^{4} \frac{b_{n}}{2} .
\end{gathered}
$$

With all these small results we finally obtain

$$
\begin{aligned}
F & =\lim _{n \rightarrow \infty} 4^{n-1} A_{n} \sin 3 \alpha_{n} \\
& =\lim _{n \rightarrow \infty} 4^{n-1} A_{n} \sin \alpha_{n}\left(4 \cos ^{2} \alpha_{n}-1\right) \\
& =\lim _{n \rightarrow \infty} 4^{n-1} A_{n} \sin \alpha_{n}\left(2 \operatorname{th}^{2} \frac{b_{n}}{2}+\operatorname{th}^{4} \frac{b_{n}}{2}\right) \\
& =\lim _{n \rightarrow \infty} 4^{n-1} A_{n} \sin \alpha_{n}\left(\frac{2}{4^{n-1}} \operatorname{th}^{2} \frac{b_{1}}{2}+\frac{1}{\left(4^{n-1}\right)^{2}} \operatorname{th}^{4} \frac{b_{1}}{2}\right) .
\end{aligned}
$$

Now we use $\lim _{n \rightarrow \infty} \sin \alpha_{n}=\sin \frac{1}{3} \pi=\frac{1}{2} \sqrt{3}$ and $\lim _{n \rightarrow \infty} A_{n}=1$ and get

$$
\begin{aligned}
F & =\lim _{n \rightarrow \infty} 4^{n-1} \cdot 1 \cdot \frac{1}{2} \sqrt{3}\left(\frac{2}{4^{n-1}} \cos ^{2} \varphi_{1}+\frac{1}{\left(4^{n-1}\right)^{2}} \cos ^{4} \varphi_{1}\right) \\
& =\lim _{n \rightarrow \infty} \frac{1}{2} \sqrt{3}\left(2 \cos ^{2} \varphi_{1}+\frac{1}{4^{n-1}} \cos ^{4} \varphi_{1}\right) \\
& =\sqrt{3} \cos ^{2} \varphi_{1}=\sqrt{3}\left(\frac{x_{1}}{h}\right)^{2} .
\end{aligned}
$$

With $x_{1}=r, h=2 r$ we get $F=\frac{1}{4} \sqrt{3}$ and $V\left(E_{0}\right)=\frac{1}{2} F=\frac{1}{8} \sqrt{3}$.

The volume $V\left(E_{1}\right)$ of the horoball sector $E_{1}$

Finding $V\left(E_{1}\right)$ we use again a trick. We perform a $H$-reflection in the $H$-plane through $M_{2}, M_{3}$ and $A_{1}$ (look at Figure 14 ). The corresponding $E$-sphere has center $M_{1}$ and radius $2 r$. Doing so, the horoball sector $E_{0}$ is mapped to $E_{1}$. $H$-reflection preserves the volume. Therefore we get $V\left(E_{1}\right)=V\left(E_{0}\right)$.

In exactly the same way we obtain $V\left(E_{0}\right)=V\left(E_{2}\right)=V\left(E_{3}\right)$. 


$$
\text { "zeitler2" — 2005/2/16 — 19:56 — page } 228 \text { — \#20 }
$$

The density

Using all our partial results it turns out that

$$
d=\frac{4 \cdot V\left(E_{0}\right)}{V(D)}=\frac{4 \cdot \frac{1}{8} \sqrt{3}}{\frac{3}{4} \sqrt{3} A}=\frac{2}{3 A} .
$$

\section{Conclusion}

\subsection{A quick summary}

We saw that the maximal packing density given in $\mathbb{H}^{2}$ and conjectured in $\mathbb{H}^{3}$ by L. Fejes Tóth is reached with special arrangements of horodisks respectively horoballs. Concerning the method we tried to keep alive the Poincaré model.

\subsection{What about didactics?}

A special problem was investigated in $\mathbb{H}^{2}$ and then in total analogy extended to $\mathbb{H}^{3}$. Doing so, a lot of interesting things was discovered by students. In this way mathematical research really develops. Now it's obvious to take the next step to $\mathbb{H}^{4}$ and even to $\mathbb{H}^{n}, n \in \mathbb{N} \backslash\{1\}$.

From this view our work has a very strong didactical component. It's a didactical paper but not at all a paper about didactics.

\section{References}

[1] J. Böhm, Simplexinhalt in Räumen konstanter Krümmung beliebiger Dimension, Journal für die reine und angewandte Mathematik 202 (1959), 15-51.

[2] H. Dörrie, Unendliche Reihen, München, 1951.

[3] H. S. M. Coxeter, The functions of Schläfli and Lobachevsky, The quaterly journal of mathematics, Oxford series 6[21] (1935), 13-29.

[4] E. P. Baranovsky, Packings, coverings, partitionings and certain other distributions of space of constant curvature, in: R. V. Gamkrelidze, Progress in mathematics $\mathbf{9}$, New York (1971), 209-253.

[5] K. Böröczky and A. Florian, Über die dichteste Kugelpackung im hyperbolischen Raum, Acta Mathematica Academiae Scientiarum Hungaricae 15 (1964), 237-245.

[6] H. Liebmann, Nichteuklidische Geometrie, Leipzig, 1905.

[7] N. J. Lobatschefskii, Zwei geometrische Abhandlungen, Leipzig, 1898. 


$$
\text { "zeitler2" — 2005/2/16 — 19:56 — page 229 — \#21 }
$$

[8] L. Fejes Tóth, On close packings of spheres in spaces of constant curvature, Publicationes Mathematicae Debrecen (1953), 158-167.

[9] L. Fejes Tóth, Lagerungen in der Ebene, auf der Kugel und im Raum, Berlin, 1953.

[10] L. Fejes Tóth, Neuere Ergebnisse in der diskreten Geometrie, Elemente der Mathematik 15 (1960), 25-36.

[11] L. Fejes Tóth, Reguläre Figuren, Budapest, 1965.

[12] I. Vermes, Ausfüllungen der hyperbolischen Ebene durch kongruente Hyperzykelbereiche, Periodica Mathematica Hungarica 10 (1979), 217-229.

[13] I. Vermes, Über reguläre Überdeckungen der Bolyai Lobatscheskischen Ebene durch kongruente Hyperzykelbereiche, Periodica Polytechnica, Mechanical Engeneering 25 (1981), 249-261.

[14] H. Zeitler, Hyperbolische Geometrie, München, 1970.

[15] H. Zeitler, Eine regulär Horozykelüberdeckung der hyperbolischen Ebene im Poincaré Modell, Elemente der Mathematik 19 (1964), 73-77.

[16] H. Zeitler, Eine reguläre Horosphärenüberdeckung des hyperbolischen Raumes, Elemente der Mathematik 20 (1965), 73-79.

[17] H. Zeitler, Über die Volumenmaßfunktion hyperbolischer Tetraeder, Der Mathematikunterricht 15 (1969), 79-97.

H. ZEITLER

BAYREUTH

GERMANY

(Received October, 2003) 